\title{
Innovation in neurosurgery
}

\author{
Mario Ammirati ${ }^{1}$ (D) \\ Received: 16 July 2017 / Accepted: 19 July 2017 /Published online: 16 August 2017 \\ (C) Springer-Verlag GmbH Austria 2017
}

The paper by Muskens et al. [1] endeavors to evaluate two innovative neurosurgical procedures, the endonasal approach to skull base meningiomas (EEMS) and the WovenEndobridge (WEB device) for endovascular treatment of intracranial aneurysms, according to the IDEAL recommendations. The IDEAL recommendations are an evolving set of guidelines on how to introduce innovations in surgery and, if widely accepted, may represent a quantum leap improvement in the way surgical innovations are introduced. This is sorely needed to improve patient safety and resource allocations. The IDEAL contribution rests, in addition and concurrently to the structured process it outlines, on ethical approval and informed consent. This diad, together with independent outcome evaluation, tries to place some constraints on potential freewheeling behavior.

Unfortunately, none of the EEMS papers evaluated come even close to the IDEAL process because of the lack of informed consent, ethical approval and outside evaluation of results.

On the WEB device side, the landscape is slightly better because there are four studies with ethical approval and patient consent (references 2, 44, 57,60) with outside evaluation being present in two of them (references 57 and 60).

The authors correctly point out that "A major change in endonasal surgery was the introduction of the endoscope, in particular for pituitary adenomas."

Mario Ammirati

mammirati@mercy.com

1 St. Rita Medical Center/Mercy Health, Lima, OH, USA
However, it is also true that endoscopic pituitary surgery was introduced in a rather unsystematic way, certainly not following any structured context; hence, its benefits, true or not, remain unproven when assessed within the general framework of a tool similar to IDEAL. Indeed, the history of the introduction of endoscopic pituitary surgery could represent a case in point on why it is necessary to have an IDEAL-like process to introduce surgical innovations.

The continuous flow of innovations in neurosurgery is fundamental and vital to the field; however, when a significant procedural or device-linked shift is proposed, it is incumbent on the potential innovator to demonstrate, in a structured way, the value of the putative proposed change. While marginal improvement in surgical techniques/procedures/instrumentation clearly does not need a structured evaluation, paradigm changes such as for example endovascular treatment of aneurysms and endoscopic cranial surgery do.

In this era of instant access to information, patients need to be protected from aggressive marketing coming from institutions, practitioners, drug and device manufacturers, etc.

It is also imperative to recognize the significant pressure to which doctors are subjected to generate products, which, in the case of surgeons, are operations.

In addition, there is a general and understandable allure on the patient side because of no visible scar and minimally invasive and technology-loaded surgery.

These two considerations, pressure to produce and public sentiment, may constitute a powerful and dangerous (for the patients and for society at large) combination.In this context, the IDEAL recommendations are vital and 
are a sensible set of guidelines to make sure that data, which are as robust as possible, are produced and that sound innovation is fostered while pseudo-innovation is rejected, at least by the medical community at large, and this is all we can do. When a structured evaluation of putative new procedures is missing, and especially when ethical approval and informed consent are lacking, it is the whole construct of meaningful innovation that suffers, being open to a potential host of often self-serving influences. In the end, it is the patient who suffers the most from the lack of structured evaluation of surgical innovation.

\section{References}

1. Muskens IS, Diederen SJH, Senders JT, Zamanipoor Najafabadi AH, van Furth WR, May AM, Smith TR, Bredenoord AL, Broekman MLD (2017) Innovation in neurosurgery: less than IDEAL? A systematic review. Acta Neurochir. doi:10.1007/s00701-017-3280-3 